\title{
AC Losses in Bi-2223 Tapes and in the 1-kA Transmission Line Model
}

\author{
L. M. Fisher, A. V. Kalinov, S. E. Savel'ev, and I. F. Voloshin \\ All-Russian Electrical Engineering Institute \\ Moscow, Russia \\ RECEIVED \\ SEP 21 1999 \\ P. Haldar \\ Intermagnetics General Corporation, Latham, NY 12110, U.S.A. \\ U. Balachandran \\ OSTI \\ Argonne National Laboratory, Argonne, IL 60439, U.S.A.
}

\begin{abstract}
The submitted manuscript has been created by the University of Chicago as Operator of Argonne National Laboratory ("Argonne") under Contract No. W-31-109-ENG-38 with the U.S. Department of Energy. The U.S. Government retains for itself, and others acting on its behalf, a paid-up, nonexclusive, irrevocable worldwide license in said article to reproduce, prepare derivative works, distribute copies to the public, and perform publicly and display publicly, by or on behalf of the Government.
\end{abstract}

\begin{abstract}
We present here results of our study of the $5-\mathrm{m}$ long AC transmission line model with $1 \mathrm{kA}$ current capability at $77 \mathrm{~K}$. Primary attention was paid to the current characteristics and $\mathrm{AC}$ losses in individual tapes and in the core of the cable. The losses were measured as a function of $\mathbf{A C}$ magnetic field amplitude in various orientations of magnetic field with respect to the plane of the tape and filaments. Hysteresis losses were close to the losses in the $\mathrm{AC}$ regime, meaning that eddy current losses in tapes may be neglected when compared with hysteresis losses. We designed and constructed a 5-m-long model of the AC transmission line using multifilamentary Bi-2223 tapes. The current core of the model contains 120 tapes for the forward line and 120 tapes for the backward line. The $A C$ losses in the current core were substantially greater than those seen in the individual tapes. The reason for this is related to a complex magnetic field distribution inside the current core.
\end{abstract}

\section{INTRODUCTION}

Many applications have been envisioned for hightemperature (high- $\mathrm{T}_{\mathrm{c}}$ ) superconductors in the electric power industry. However, the critical current of today's high- $T_{C}$ tapes strongly depends on magnetic field. Therefore, the most attractive application of high- $T_{c}$ superconductivity is in electric power cables, where the superconductor operates in a relatively low magnetic field. Prototypes of $77-\mathrm{K}$ cables have been designed, constructed, and tested with $\mathrm{Bi}-2223 / \mathrm{Ag}$ superconducting tapes (see, for example, Refs. 1-5). Highvoltage and current input problems [2,3], mechanical degradation and cooling processes [2-4], current capability, and $A C$ losses $[1,3,5]$ have been studied. However, most of the published papers are devoted to studies of single tape characteristics or to the design and testing of some type of transmission line models. Data obtained for the single tapes cannot, however, be used directly to estimate the parameters of such a complicated unit as an AC transmission line [6,7], because the line consists of many (usually hundreds) of tapes combined in a certain manner. The current distribution, the magnetic field dependence of the critical current, and non-

Manuscript received September 15. 1998.

This work was supported by the U.S. Department of Energy (DOE), Energy Efficiency and Renewable Energy, as part of a DOE program to develop electric power technology, under Contract W-31-109-Eng-38; and by the DOE, Office of Arms Control and Nonproliferation (NN-40), Russia/NIS Nuclear Materials Task Force Office, through its New Independent States Initiatives for Proliferation Prevention (NIS-IPP) Program. under Contract IPP-ANL-033-RS. equivalent positions of the tapes in the line may drastically affect the full losses in a transmission line, and the losses will not be just the sum of the losses in single tapes.

In this study, we investigated $\mathrm{AC}$ losses in single $\mathrm{Bi}$ 2223/Ag tapes and in 5-m-long AC transmission line model. We designed, constructed, and tested the $\mathrm{AC}$ transmission line model. The model contains 120 forward current carrying tapes and 120 return current carrying tapes. The capability of the transmission line current was tested for currents up to 1200 A (RMS).

\section{V-I CHARACTERISTICS AND AC LOSSES IN Bi-2223 TAPES}

All studies were performed on a $\mathrm{Bi}-2223 / \mathrm{Ag}$ tape that was fabricated by Intermagnetics General Corporation. This tape is $4.4 \mathrm{~mm}$ wide and $0.19 \mathrm{~mm}$ thick and consists of 37 filaments. The critical current of an elementary tape was measured by the conventional four-point-probe method. V-I characteristics of several tapes, each $\approx 50 \mathrm{~cm}$ long (cut from a long-length tape) in a DC regime, were measured by a nanovoltmeter (Keithley-181). To realize the field configuration close to the transmission line, we prepared the sample as two strips put together (like direct and back line wires) with a thin insulating layer between them and soldered at one end. On the other end, two current leads were soldered. To check the homogeneity of the current capability, we mounted four voltage contacts (two contacts for each strip). The distance between contacts was $365 \mathrm{~mm}$ for each pair. An increase in voltage with an increase in current at $I \approx 15$ and 20 A for a pair of strips was observed. At higher currents, the voltage increased sharply as a power function of 1 . The exponents were $\approx 25$ and 15 for the two strips. At I $>30 \mathrm{~A}$, the exponents decreased. According to the conventional criterion $(1 \mu \mathrm{V} / \mathrm{cm})$, the critical currents are 26 and $22 \mathrm{~A}$.

\section{A. Measurement of Losses by Magnetization Methods \\ 1. DC Magnetic Field}

Samples for the DC magnetization measurements were accurately cut from a $\mathrm{Bi}-2223 / \mathrm{Ag}$ tape that contained 37 superconducting filaments. Each sample consisted of 10 pieces of tape measuring $0.5 \times 0.44 \times 0.019 \mathrm{~cm}^{3}$. The pieces were combined with insulating tape, so they were insulated 


\section{DISCLAIMER}

This report was prepared as an account of work sponsored by an agency of the United States Government. Neither the United States Government nor any agency thereof, nor any of their employees, make any warranty, express or implied, or assumes any legal liability or responsibility for the accuracy, completeness, or usefulness of any information, apparatus, product, or process disclosed, or represents that its use would not infringe privately owned rights. Reference herein to any specific commercial product, process, or service by trade name, trademark, manufacturer, or otherwise does not necessarily constitute or imply its endorsement, recommendation, or favoring by the United States Government or any agency thereof. The views and opinions of authors expressed herein do not necessarily state or reflect those of the United States Government or any agency thereof. 


\section{DISCLAIMER}

Portions of this document may be illegible in electronic image products. Images are produced from the best available original document. 
from each other, and the resulting sample was approximately a cube.

A vibrating-sample magnetometer was used to measure the magnetization loops $M(H)$. Measurements were performed at liquid nitrogen temperature in magnetic fields up to $1 \mathrm{kOe}$. Three DC magnetic field orientations were considered: 1 - field parallel to the tape surface but perpendicular to the filaments, 2 - field directed along the filaments, and 3 - field perpendicular to the plane of the tape.

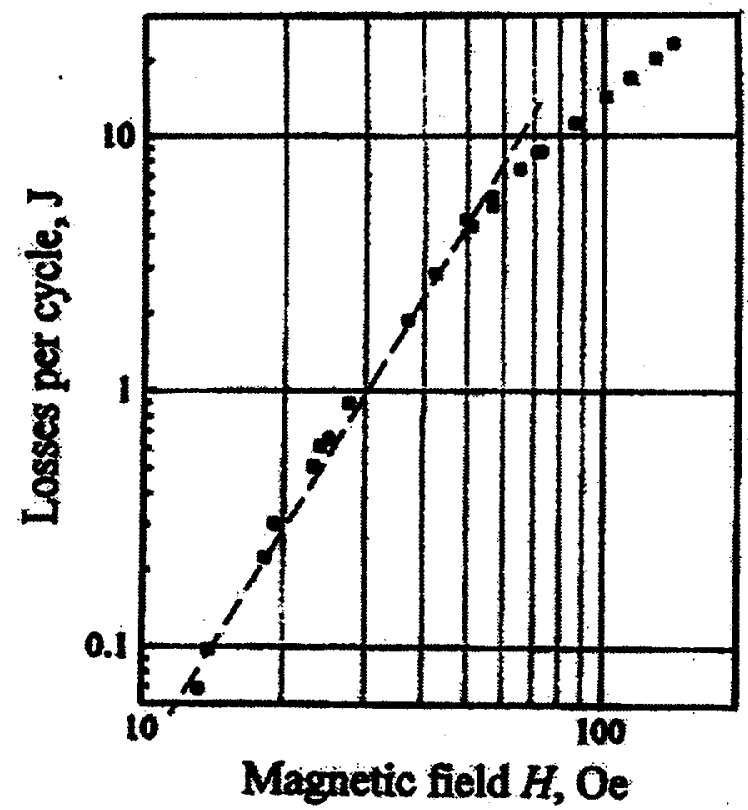

Fig. 1. Hysteresis losses per cycle per cubic meter as a function of magnetic field H. Dashed line corresponds to cubic relationship.

Magnetization loops for the three orientations show that the areas defined by field orientations 1 and 2 are similar to each other, whereas the area defined by 3 is much greater [8]. This difference may be related to the orientation of our experiment, in particular, with the demagnetization factors caused by the flat form of the filaments. Hysteresis losses were determined from the area of the magnetization loops for Orientation 1 in a DC regime. The dependence of hysteresis losses $\mathrm{P}_{\mathrm{H}}$ on the maximum $\mathrm{H}$ per cycle is presented in Fig. 1. This dependence is typical for hard superconductors. At low fields of $\mathrm{H}<50$ Oe, losses are proportional to $\mathrm{H}^{3}$ (dashed line in the figure). At higher fields, the exponent tends to be unity.

\section{AC Magnetic Field}

To define the total losses in the tape, we measured the dependence of the imaginary part $\chi$ " of the AC susceptibility as a function of the magnitude $h_{0}$ of the $A C$ magnetic field for two directions of $h$ with respect to the filaments. The losses $Q$ (in Watt) are related to $\chi^{\prime \prime}$ by the simple equation, $Q=10^{-7} \chi^{\prime \prime} h_{o}{ }^{2} \mathrm{Vf} / 4$, where $V$ is the sample volume in $\mathrm{cm}^{3}$. The dependence on AC field amplitude of the total losses per $1 \mathrm{~m}$ of tape length for two directions of applied magnetic field are shown in Fig. 2. Curve 1 in the figure corresponds to $h$ parallel to the filaments and Curve 2 was obtained for $h$ perpendicular to the filaments.

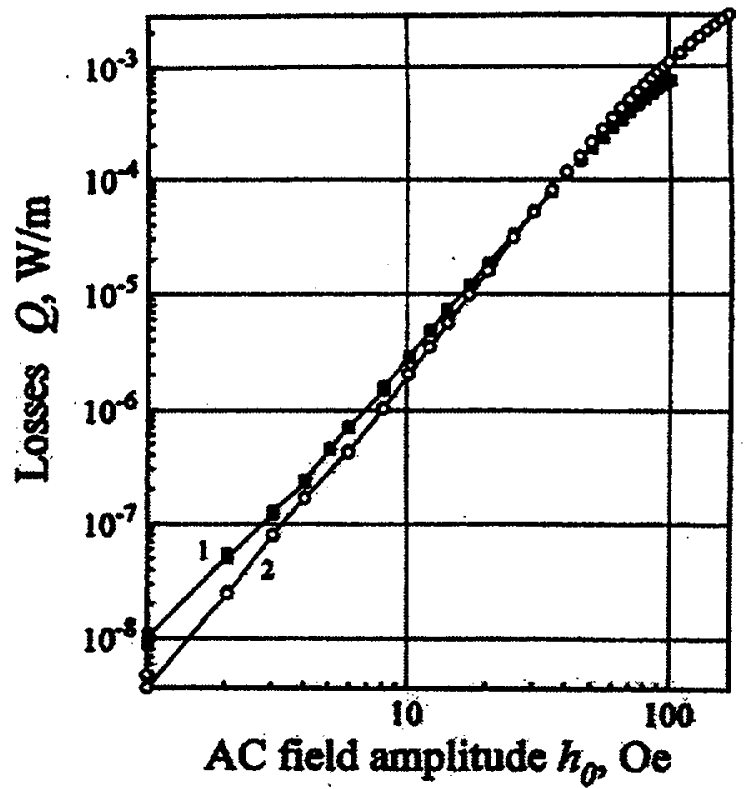

Fig. 2. AC losses for orientations where $A C$ field amplitude $h_{0}$ is parallel to filaments (Curve 1) and perpendicular to them (Curve 2).

\section{B. Measurement of Losses by AC Current Methods}

To model conditions similar to those of a transmission line, we studied the AC transport properties of the doublestrip sample. The V-I characteristics of the sample were measured for the AC current: $I=I_{0} \cos (w t), \omega / 2 \pi=f$, at frequencies of 13 and $60 \mathrm{~Hz}$. A lock-in amplifier PAR 124A was used in differential mode to detect the voltage that was in phase with the current and shifted on $\pi / 2$ with respect to it. The reference was a signal taken from a noninductive resistor in series with the superconducting tape.

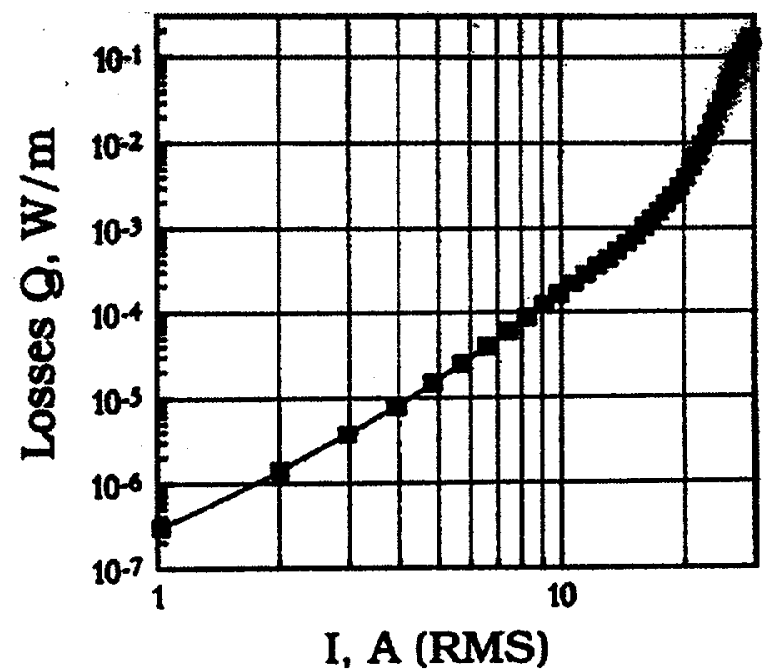

Fig. 3. AC losses of a tape at $60 \mathrm{~Hz}$ as a function of RMS AC current. 
A drop in the $\mathrm{AC}$ voltage is defined by the transport resistivity (at high currents), the remagnetization losses, and the eddy current losses in a normal metal. The losses were calculated by using the in-phase voltage drop $\mathrm{V}$ as follows: $\mathrm{Q}=\mathrm{V} \times \mathrm{I}$ (Fig. 3).

In the previous section, we have considered the data for losses obtained by three methods. All of the data are in good agreement over the entire field range presented. The field range limit corresponds to $\approx 1 / 2$ of the critical current value, i.e., the typical operation range of the transmission line current. The results from DC and AC magnetization methods correlate well. Differences are more pronounced at high currents. Indeed, if some portions of the measured tape contained bad clusters, the losses should be higher in these regions of the tape than in other homogeneous ones and thus lead to an increase in losses, which was observed in our experiment.

\section{TESTING THE MODEL AND DEFINING ITS CHARACTERISTICS}

To construct the model, we used $\mathrm{Bi}-2223 / \mathrm{Ag}$ tape produced by Intermagnetics General Corporation. The total length of these tapes was $\approx 1200 \mathrm{~m}$ and they displayed an average critical current of $\approx 8 \mathrm{~A}$. We also constructed a 5-mlong transmission line current core with a current capability of up to $1 \mathrm{kA}$.

\section{A. Current Core Construction}

We divided the material into forward and backward lines according to material volume. The lines contained $1205-\mathrm{m}$ tapes. To achieve a good filling coefficient, we designed the following current core. Using a stainless steel core $(40 \mathrm{~mm}$ in diameter) as a former of the cable, we divided tapes into 12 groups (20 tapes in each) so that the current capability of each strand of the cable was almost the same $(\approx 160 \mathrm{~A})$.. We used a 50-mm Mylar tape as a base of each strand. Each tape in the strand was freely inserted into slits cut across the Mylar tape. The width of the slits was $\approx 120 \mathrm{~mm}$. Adjacent tapes were placed on opposite surfaces of the Mylar tape. The strand exhibited good rigidity and each superconducting tape in the strand was well spaced and electrically isolated from other tapes.

Wire transposition in the current core does not significantly alter the performance of transmission cables $[9,10]$. In our model, each pair of strands was put on a thin Mylar tape in an X-form, wound on the stainless steel former. Such a construction enabled the transposition of the tapes in two strands, but the adjacent three pairs of strands were not transposed. The outer diameter of the current core after winding was $\approx 48 \mathrm{~mm}$. In accordance with the superconducting current capability, we expected to get a total line critical current of $\approx 160 \times 6=960 \mathrm{~A}$.

\section{B. Electrical Circuit and Regime of Testing}

To test the transmission line model, we put the current core into the cryogenic envelope. We used the short-circuit testing regime by connecting all of the flexible copper wires from one end of the current core. The current and potential wires from the other end were inserted through a cap on the cryogenic envelope into the testing circuits. All flexible current wires were electrically isolated.

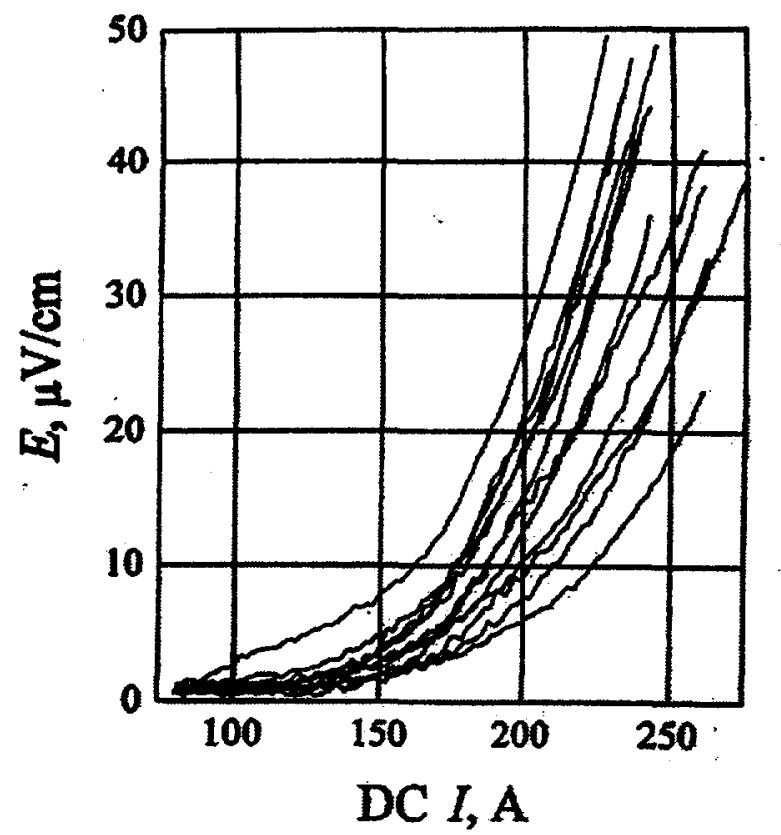

Fig. 4. V-I characteristics of tape.

We used a transformer current input to supply current to the core. The primary winding $\mathrm{L}_{\mathrm{p}}$ of the transformer was connected to the output of a power amplifier with a current feedback. The transformer ratio was 12 . AC frequency was adjusted by an internal oscillator in the lock-in amplifier PAR 124A. The secondary winding $L_{S}$ was connected to the current core of the model. The value of the current was measured by the current transformer $\mathrm{L}_{\mathrm{I}}$. To measure AC losses in the transmission line, the potential probes were connected to the input of the selective amplifier PAR 124A. The copper coil $\mathrm{Lim}$ was used for phase adjustment in the lock-in. This copper coil was arranged on the surface of the current lead in such a way that the magnetic field of the current was parallel to the axis of the coil. Hence, the voltage in the $\mathrm{L}_{\mathrm{im}}$ coil was out of phase with the current and it was used for phase tuning in the lock-in detector, and to compensate for the out-of-phase (inductive) component in the signal that permitted us to reduce the phase separation error.

The measurements were performed at liquid nitrogen temperature. The current core was immersed in liquid nitrogen. The system was cooled by liquid nitrogen from a tank at a pressure of $\approx 0.5$ atm and cooling time was $\approx 1 \mathrm{~h}$.

\section{Line Model Testing}

\section{Testing Current Capability of Strands in DC Regime}

In the first stage of testing, we determined the DC V-I characteristics of individual strands. DC current was supplied to neighboring strands in series, and the voltage drop on each strand was measured by a Keithley 181 nanovoltmeter. Figure 4 shows the V-I characteristics of all strands. Despite matching the equal current capability of 
strands, dispersion is noticeable. Contact resistivity changed from one strand to another and is in the range of 110 to 120 $\mu \Omega$.

\section{Testing the Line Model Current Core in AC Regime}

We tested the current core of the transmission line model in the $\mathrm{AC}$ regime at $60 \mathrm{~Hz}$. The control voltage of power amplifier PAR 124A, which fed the primary winding of the transformer, was approximately sinusoidal. A superharmonic at the testing regimes we used did not exceed 5\%. The inductance of the current core with the copper current leads was $\approx 0.3 \mathrm{mH}$. The component that was in phase with the current (active voltage), in particular, the voltage drop on the copper current leads, was linear with the current. To obtain the active voltage that was related to the superconducting current core, we subtracted the linear part of the voltage drop. The dependence $V(I)$ is close to a power function with the exponent $\mathrm{n}=3.12$. Figure 5 (plotted in a $\log -\log$ scale) shows the dependence of $\mathrm{AC}$ losses $\mathrm{W}$ in the current core as a function of the transport current $I$. The experimental results are shown by closed circles. The losses $W$ increased with I proportional to $\mathrm{I}^{\mathrm{n}}$, with $\mathrm{n}=3.8$ at currents $\mathrm{I}<700 \mathrm{~A}$. At higher currents, losses increased faster. Such a behavior is related to the transition to the resistive state.

Thus, the AC transmission line model can carry an AC transport current of up to $1000 \mathrm{~A}$ at liquid nitrogen temperature. An increase in the current from zero to $1100 \mathrm{~A}$ does not lead to serious disturbances in loading characteristics of the superconducting current core.

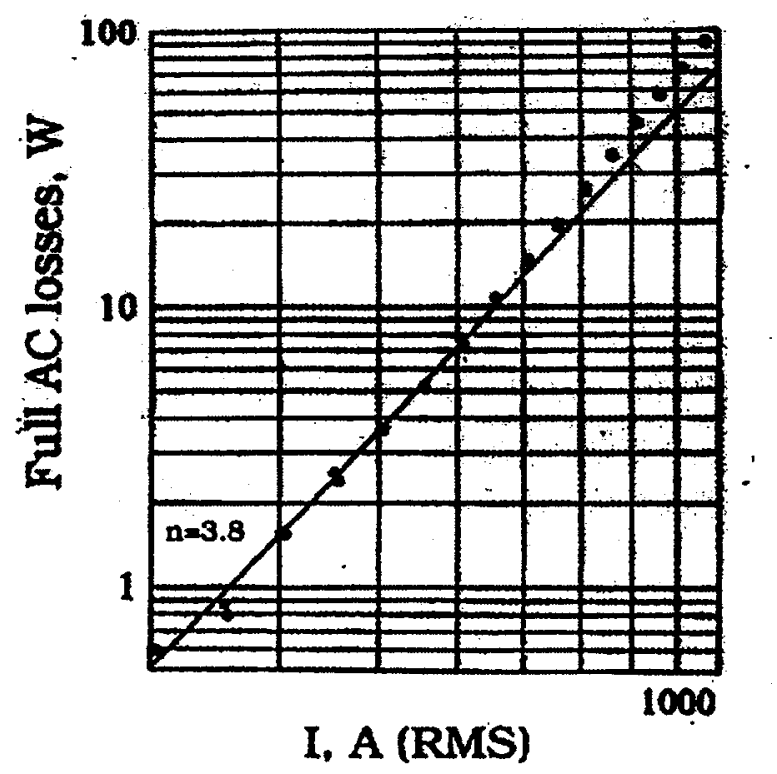

Fig. 5. Full $\mathrm{AC}$ losses in line model (points) and fit function that corresponds to data in a low-current region (solid line).

\section{CONCLUSIONS}

The experimental results reported in this paper were obtained during our study of an AC transmission line model. The current core of the model was constructed from $\mathrm{Bi}$ $2223 / \mathrm{Ag}$ tapes that were produced by Intermagnetics General Corporation. In the initial stage, the critical current capability and the AC losses were investigated. The losses were measured as a function of DC and AC magnetic field amplitude for various orientations with respect to the plane of the tape and filaments. Hysteresis losses are shown to be close to the losses in the AC regime. This finding means that eddy current losses in tapes may be neglected when compared with hysteresis losses.

We designed and constructed a model of the AC transmission line using these $\mathrm{Bi}-2223 / \mathrm{Ag}$ tapes. The current core of the model contains 120 tapes for the forward line and 120 tapes for the backward line (each $5 \mathrm{~m}$ long). Expected critical current was $\approx 960 \mathrm{~A}$.

Our tests of this model showed that the current capability of the current core correlates well with data obtained for single tapes. The AC losses in the current core are shown to be substantially greater than those shown by the data for the individual tapes. The reason for this is related to a complex magnetic field distribution inside the current core.

\section{REFERENCES}

[1] T. Hara, H. Ishii, and S. Honjo 1996 Proc. 16th Intl. Cryogenic Engineering Conf./Intl. Cryogenic Materials Conf., Kitakyushu, Japan, May 20-24, 1996 eds. T. Haruyama, T. Mitsui, K. Yamafuji (Elsevier, Oxford, UK, 1997) Part 2, p. 963.

[2] T. Shibata et al. 1996 Proc. 16th Intl. Cryogenic Engineering Conf./ntl. Cryogenic Materials Conf., Kitakyushu, Japan, May 20-24, 1996 eds. T. Haruyama, T. Mitsui, K. Yamafuji (Elsevier, Oxford, UK, 1997) Part 2, p. 967.

[3] T. Masuda et al. 1996 Proc. 16th Intl. Cryogenic Engineering Conf./Intl. Cryogenic Materials Conf., Kitakyushu, Japan, May 20-24, 1996 eds. T. Haruyama, T. Mitsui, K. Yamafuji (Elsevier, Oxford, UK, 1997) Part 2, p. 971.

[4] J. Fujikami et al. 1996 Proc. 16th Intl. Cryogenic Engineering Conf./Intl. Cryogenic Materials Conf., Kitakyushu, Japan, May 20-24, 1996 eds. T. Haruyama, T. Mitsui, K. Yamafuji (Elsevier, Oxford, UK, 1997) Part 2, p. 975.

[5] S. Mukoyama et al. 1996 Proc. 16th Intl. Cryogenic Engineering Conf./Intl. Cryogenic Materials Conf., Kitakyushu, Japan, May 20-24, 1996 eds. T. Haruyama, T. Mitsui, K. Yamafuji (Elsevier, Oxford, UK, 1997) Part 2, p. 979.

[6] S. Fleshler et al. Appl. Phys. Lett. 1995673189.

[7] D. E. Daney et al. IEEE Trans. Appl. Supercond. 19977310.

[8] L. M. Fisher, A. V. Kalinov, S. E. Savel'ev, I. F. Voloshin, P. Haldar, and $U$. Balachandran. Superconductor Science and Technology (1998), submitted.

[9] L. M. Fisher et al. Phys. Rev. B, 19924610986.

[10] D. Eckert, G. Enderlein, F. Lange IEEE Trans. Magn. 1974 MAG-11 377. 